\title{
Influence of plasticizer structure on stability of polystyrene scintillator optical characteristics
}

\author{
V.G. Senchishin, A.Yu. Borisenko, V.D. Titskaja, V.S. Koba, V.N. Lebedev, A.F. Adadurov*, \\ V.N. Osadchenko \\ Institute for Single Crystals, National Academy of Science of Ukraine, 60 Lenin Ave., 310001 Kharkiv, Ukraine, \\ tel.(0572)307969,E-mail: sench@isc.kharkov.com \\ *Kharkov State University, sq. Svobody 4, 310077 Kharkiv, Ukraine
}

\begin{abstract}
The radiation and thermal stability of optical characteristics of polystyrene scintillators modified by plasticizers based on derivatives of naphthalene, ditolylmethylene, diphenylene oxide, ether of phthalic acid and oligo-phenyl-methyl-siloxanes were studied. It was shown that structure and size of plasticizer molecule make a definite influence on scintillators characteristics of operational conditions stability.
\end{abstract}

Keywords: polystyrene scintillator, radiation stability, thermostability, plasticization.

Paper received 04.11.00; revised manuscript received 19.01.01; accepted for publication 16.02.01.

\section{Introduction}

The polystyrene scintillator (PS) is the radioluminophor used in scitillator detectors for detection and characterizing measurements of ionizing radiation: $\alpha, \beta, \gamma$, neutrons and others. PS converts an energy of ionizing radiation into light flashes - scintillations which are collected by photomultiplier $[1,2]$.

During operational time, the main features of scintillators - scintillation efficiency and transparency to proper luminescence light - become worse as a result of radiation destruction and material aging.

To improve the radiation stability, it was offered to use plasticizers of different chemical nature [1,2]. Present work is aimed to investigate the influence of structure of molecules plasticizers of different chemical nature on radiation and thermal stability. As plasticizer the derivatives of naphthalene, ditolyl methylene, diphenylene oxide, ether of phthalic acid and oligo-phenyl-methyl-siloxanes of $15-20 \%$ concentrations were used.

The plasticizers which we selected for the modification of polystyrene scintillators were distinguished by the structure and size of molecule. The common feature of all used plasticizers was presence of naphthalene or phenyl radicals in their molecules that provides sufficiently greater value of dissolvability parameter of plasticizers $\delta=18-21\left(\mathrm{MJ} / \mathrm{m}^{3}\right)^{1 / 2}$. The dissolvability parameter of plasticizers that characterizes cohesion forces of molecular interaction is close to value of polystyrene dissolvability parameter $\delta=19\left(\mathrm{MJ} / \mathrm{m}^{3}\right)^{1 / 2}$, which ensures sufficiently good compatibility of plasticizers with polystyrene matrix of PS. The parameter of dissolvability is defined by the expression [3]:

$$
\delta=\frac{d \cdot \sum G}{M}
$$

where $G$ are attraction constants of separate groups of molecule; $d$ is a density; $M$ is a molecular mass of plasticizer.

\section{Experiment}

Samples of scintillators were produced by thermal polymerization of styrene with scintillation additives (PTP $2 \%$, POPOP $0 / 02 \%$ ) and plasticizers, as well as by the method of injection molding. The samples were made in the form of cylinders of $30 \mathrm{~mm}$ height and $16 \mathrm{~mm}$ diameter.

Transparency measurement was executed using a laser on cadmium vapours $(\lambda=441 \mathrm{~nm})$. The intensity of light falling $I_{0}$ and passed $I$ through the sample of a length $x$ was measured. Transparency $\left(L_{0}\right)$ was calculated from the expression:

$$
I=I_{0} \cdot \xi \cdot \exp \left(-x / L_{0}\right)
$$

where $\xi$ is a factor taking into account the reflection of light from the surface of PS sample. 


\section{V.G. Senchishin et al.: Influence of plasticizers structure on stability of ...}

To study PS radiation stability, the samples were irradiated to the dose of $40 \mathrm{kGy}$ by $\gamma$-radiation from a ${ }^{60} \mathrm{Co}$ source in air at the $20{ }^{0} \mathrm{C}$ temperature. $\gamma$ - radiation power was $0.1-0.25 \mathrm{kGy} /$ hour. The transparency changing was estimated by the following parameter $k$ :

$k=\frac{L_{0}-L}{L_{0}} \cdot 100$

where $L_{0}, L$ are transparencies before and after irradiation, respectively.

Samples with $k=0$ are characterized by the highest radiation stability, greater value of $k$ corresponds to lower radiation stability.

To predict behavior of PS material stability with time, the thermal aging of samples was studied. Samples were heated in the air thermostat under $70^{\circ} \mathrm{C}$ within 24 hours. Masses of samples were measured before and after heating, and the plasticizers migration rate was determined in accord with [4]:

$$
\mu=\frac{m_{0}-m}{m_{0}} \cdot 100
$$

where $m_{0}$ is a mass of sample before, and $m$ after heating.

Using $50^{\mathrm{x}}$ optical microscope MIM-7, we observed changes occurring in the volume and on surfaces of samples.

\section{Results}

After irradiation, PS samples in yellow and brown colours simultaneously with significant changes of trans- parency were observed. This is the result of formation in PS volume radiation induced radicals that absorb the luminescence light of PS.

Fig. 1a presents the relative PS transparency $k$ vs the parameters of plasticizers: molecular mass $M$ and dissolvability parameter $\delta$. It is worth to note, that the modification of PS by derivatives of naphthalene, ditolyl methylene, diphenylene oxide, increases the radiation stability of PS optical features $(k \leq 20 \%)$. While the use of ether of phthalic acid and oligo-phenyl-methyl-siloxanes leads to greater changes of transparency, $k \geq 30 \%$ (smaller radiation stability).

The PS samples radiation stability increases with growing naphthalene and phenyl radical parts in plasticizer molecules. Thus, according to Fig. 1a, increasing of dissolvability parameter value $\delta$, naphthalene and phenyl radicals part of the molecule, decreases relative change of transparency $k$. It means that radiation stability of PS samples increases (Fig. 1a).

The PS radiation stability is increased due to the fact that plasticizer molecules of naphthalene and phenyl radicals diffuse and dissipate the part of excitation energy absorbed by PS material, preventing the polystyrene macromolecules destruction.

The next reason of PS samples radiation stability increase and reduction of parameter $k$ is structure plasticization of PS material, efficiency of which increases with reducing plasticizer molecule sizes (i. e., at smaller $M$, Fig.1a). To provide active structured plasticization, molecule plasticizers should have the size close to that of polystyrene monomer [5]. In this case, mobilities of bulk phenyl group of monomer molecules and segments of polystyrene macromolecules are relieved. It provides an active diffusion
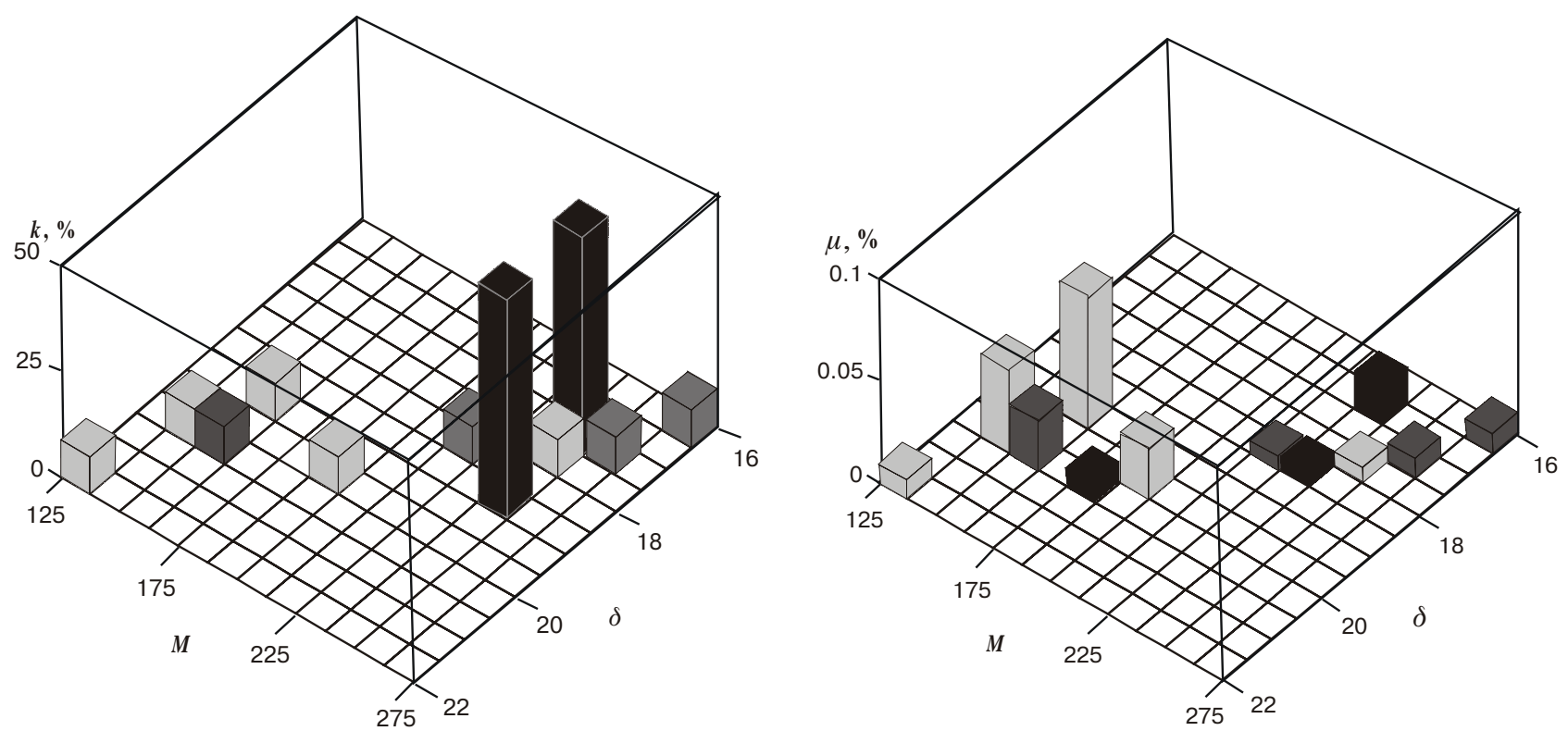

Fig. 1. The relative PS transparency changing $k$ under irradiation (a) and plasticizers migration $\mu$ under thermal aging (b) vs plasticizers molecule structures features. 


\section{V.G. Senchishin et al.: Influence of plasticizers structure on stability of ...}

of oxygen into PS volume, which enables oxidation and recombination of radiation induced radicals, that is the centers of PS luminescence light absorption. Active oxidation and recombination of radicals allow PS to recover their optical characteristics simultaneously with radiation destruction. As a result, after irradiation there is no observed profound change in transparency of plasticizer - PS modified samples.

After thermal aging, the change of PS sample mass is observed, caused by plasticizer migration. Fig. 1b shows dependences of plasticizer migration on molecular mass $M$ and dissolvability parameter $\delta$. As can be seen, increasing of plasticizer molecule size (molecular mass $M$ ) and cohesion energy (parameter of dissolvability $\delta$ ) reduce plasticizer migration. Thus, in the case of using the ether of phthalic acid and oligo-phenyl-methyl-siloxanes, the only small migration $(\mu=0.05 \%)$ is observed and transparency changes do not occur. When using derivatives of naphthalene, ditolyl methylene, diphenylene oxide, we observed the more intensive migration $(\mu \geq 0.1 \%)$ resulting in formation of microcracks and micropores, that lead to decreasing PS sample transparency.

The plasticizers migration can be lowered down to the value of $0.05 \%$ by producing the PS with high mo- lecular mass according to the technology of bulk polymerization or using the technology of injection molding.

Therefore, the use of plasticizers based on derivatives of naphthalene, ditolyl methylene, diphenylene oxide when producting PS of high molecular mass in accordance with technology of bulk polymerization or using the technology of injection molding, which enables to get PS with high stability of optical features during operational time.

\section{References}

1. Markley F., Lebedev V.N., Kovtun V.E., Koba V.S., Kuznichenko A.V., Tizkaja V.D., Budagov J.A., Bellettini G., Seminozhenko V.P., Zalubovsky I.I., Chiricov-Zorin I.E., A new radiation stable plastic scintillator // Nucl.Instr. and Meth. A364, pp. 253-257 (1995).

2. V.G. Vasil'chenko, V.G. Lapshin, A.I. Peresypkin, A.A. Konstantinchenko, A.I. Pyshev, V.M. Shershukov, B.V. Semenov, A.S. Solov'ev, New results of plasric scintillators radiation damage studies // Functional materials 3(4), pp. 541-545, (1996).

3. Encyclopedia of polymers V.1 Sovetskaja Encyclopedia, Moskva, 1972, 1224 p. (Russian).

4. GOST 14926-81 Plastics. Method for determination of plasticizers migration, in Standard Publishers, 4 p., (1981).

5. Kozlov P.V., Papkov S.P., Physical and chemical principles of polymer plasticization, Chimija, Moskva, 1982, 224 p. (Russian). 